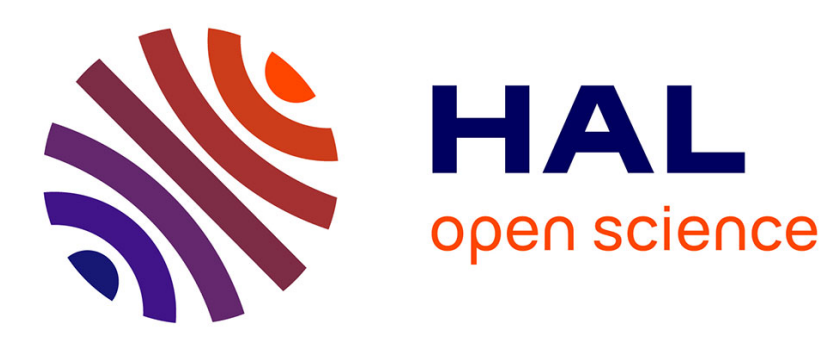

\title{
Phase Behavior and Relative Stability of Malonamide Polymorphs
}

\author{
Yohann Corvis, N. Guiblin, Philippe Espeau
}

\section{To cite this version:}

Yohann Corvis, N. Guiblin, Philippe Espeau. Phase Behavior and Relative Stability of Malonamide Polymorphs. Journal of Physical Chemistry B, 2014, 118, pp.1925-1931. 10.1021/jp409797k . hal01053342

\section{HAL Id: hal-01053342 \\ https://hal.science/hal-01053342}

Submitted on 24 Nov 2014

HAL is a multi-disciplinary open access archive for the deposit and dissemination of scientific research documents, whether they are published or not. The documents may come from teaching and research institutions in France or abroad, or from public or private research centers.
L'archive ouverte pluridisciplinaire HAL, est destinée au dépôt et à la diffusion de documents scientifiques de niveau recherche, publiés ou non, émanant des établissements d'enseignement et de recherche français ou étrangers, des laboratoires publics ou privés. 


\title{
Phase Behavior and Relative Stability of Malonamide Polymorphs
}

\author{
Yohann Corvis, ${ }^{\dagger}$ Nicolas Guiblin, ${ }^{\ddagger}$ and Philippe Espeau* ${ }^{\dagger}$ \\ †EA 4066 "Physico-Chimie Industrielle du Médicament”, Sorbonne Paris Cité, Faculté des Sciences Pharmaceutiques et Biologiques, \\ Université Paris Descartes, 4 Avenue de l'Observatoire, 75006 Paris, France \\ ${ }^{\ddagger}$ Laboratoire Structures, Propriétés et Modélisation des Solides (SPMS), CNRS UMR 8580, Ecole Centrale Paris, \\ Grande Voie des Vignes, F-92 295 Châtenay-Malabry, France
}

ABSTRACT: The three known polymorphs of malonamide have been characterized by thermal analysis and X-ray powder diffraction. The melting thermodynamic characteristics of the three forms are thus proposed in the present paper. From these data, the relative thermodynamic stability of these three solid forms has been determined. It appears that an enantiotropic behavior is established between the monoclinic and the orthorhombic phases while the quadratic one is monotropic with respect to the other two.

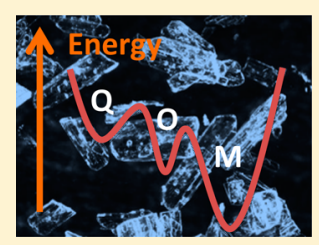

\section{INTRODUCTION}

Malonamide, otherwise known as malonodiamide, is often used, in the pharmaceutical field, as an intermediate reactant to prepare pharmacologically active derivatives. Some derivatives have been patented for the therapy of cardiovascular disorders like thromboembolic diseases or restenoses, ${ }^{1}$ and others for topical formulations to treat sebaceous gland disorders. ${ }^{2}$

A malonamide monoclinic (M) structure was reported at ambient conditions in the 1970s. ${ }^{3}$ Approximately 20 years later, two other crystalline phases (i.e., quadratic ( $Q$ ) and orthorhombic (O) phases) were reported and examined by thermal analysis. ${ }^{4}$ An enantiotropic behavior has been found between the monoclinic (M) and the quadratic (Q) phases, and a monotropic behavior has been reported for the third one. ${ }^{4}$ However, it was not before 2005 that the crystal structures of these two latter phases have been solved. ${ }^{5,6}$ Data on crystalline forms were reexamined by thermal analysis and infrared spectroscopy, confirming the enantiotropic and monotropic relationships. ${ }^{7}$ However, the monotropic behavior of a system cannot be determined solely on the basis of thermal analysis. Indeed, the fact that one cannot observe experimentally a transition does not prove that it does not exist. In the present paper, the demonstration will be made for the $\mathrm{M}$ toward $\mathrm{Q}$ malonamide stable transition. The question may also arise for the other two solid-solid phase transitions because, although not observed experimentally, these transitions can occur, even if they are metastable (nonreversible). The relative stability of the three crystalline forms has to be established in order to unambiguously prove the enantiotropic or monotropic behavior of the system over the entire temperature range. For that, calculation of the Gibbs free energies as a function of the temperature from melting data of each polymorphic form was carried out. The information deduced from the energy-temperature diagram about the relative thermodynamic stability of the solid phases, i.e., metastability versus stability, necessarily gives access to the relative stability of the transition points (solid-solid and solid-liquid). Indeed, the higher the energy of the transition point, the lower its stability.

\section{EXPERIMENTAL SECTION}

Chemicals. Malonamide (purity $\geq 97 \%$ ) was purchased from Aldrich. The product was used without further purification.

Thermal Analysis. Temperatures and enthalpies of transition were determined using a differential scanning calorimeter 822e (Mettler-Toledo, Switzerland); scan rate: $10{ }^{\circ} \mathrm{C} \cdot \mathrm{min}^{-1}$. The calibration was made using high purity indium $\left(T_{\text {fus }}=\right.$ $156.6{ }^{\circ} \mathrm{C}, \Delta_{\text {fus }} H=3.27 \mathrm{~kJ} \cdot \mathrm{mol}^{-1}$ ). Six independent measurements were carried out on heating and cooling. The errors on the enthalpies and the temperatures resulted from the calculation of the standard deviation from the average value. The molar heat capacities $\left(C_{p}\right)$ were also determined with the thermal analyzer DSC 822e (Mettler-Toledo,Switzerland). The samples were ground in a mortar beforehand-in order to minimize the interstices between particles - then weighed with a microbalance sensitive to $1 \mu \mathrm{g}$, and finally sealed in $40 \mu \mathrm{L}$ aluminum pans. To minimize the measurement errors, the pans were completely filled before being sealed. A differential scanning calorimetry (DSC) experimental procedure based on sapphire reference with a $15^{\circ} \mathrm{C} \cdot \mathrm{min}^{-1} \mathrm{scan}$ rate allowed us to obtain the evolution of the heat flow as a function of temperature. The baseline used for the signal integration was established by an isothermal run of $10 \mathrm{~min}$ before and after the dynamic run described above. The uncertainty was determined by calculating the standard deviation from the average value errors of eight independent measurements for each physical state. This method has been previously validated. ${ }^{8}$

X-ray Powder Diffraction. X-ray data collection was performed on a homemade diffractometer with a Rigaku RA$\mathrm{HF} 18$ rotating anode generator $(50 \mathrm{kV}, 300 \mathrm{~mA})$. Monochromatic Co K $\alpha_{1}(\lambda=1.78897 \AA)$ radiation was selected by means of a nickel filter. The sample was placed on a cryofurnace (TBT, Air Liquide) with a temperature range between -190 and $210{ }^{\circ} \mathrm{C}$, using liquid nitrogen. Each data scan was recorded between 5 and $60^{\circ}$ in $2 \theta$, with a step size of $0.02^{\circ}$ and a counting time of $1 \mathrm{~s}$ per step.

\footnotetext{
Received: October 2, 2013

Revised: December 20, 2013

Published: January 27, 2014
} 

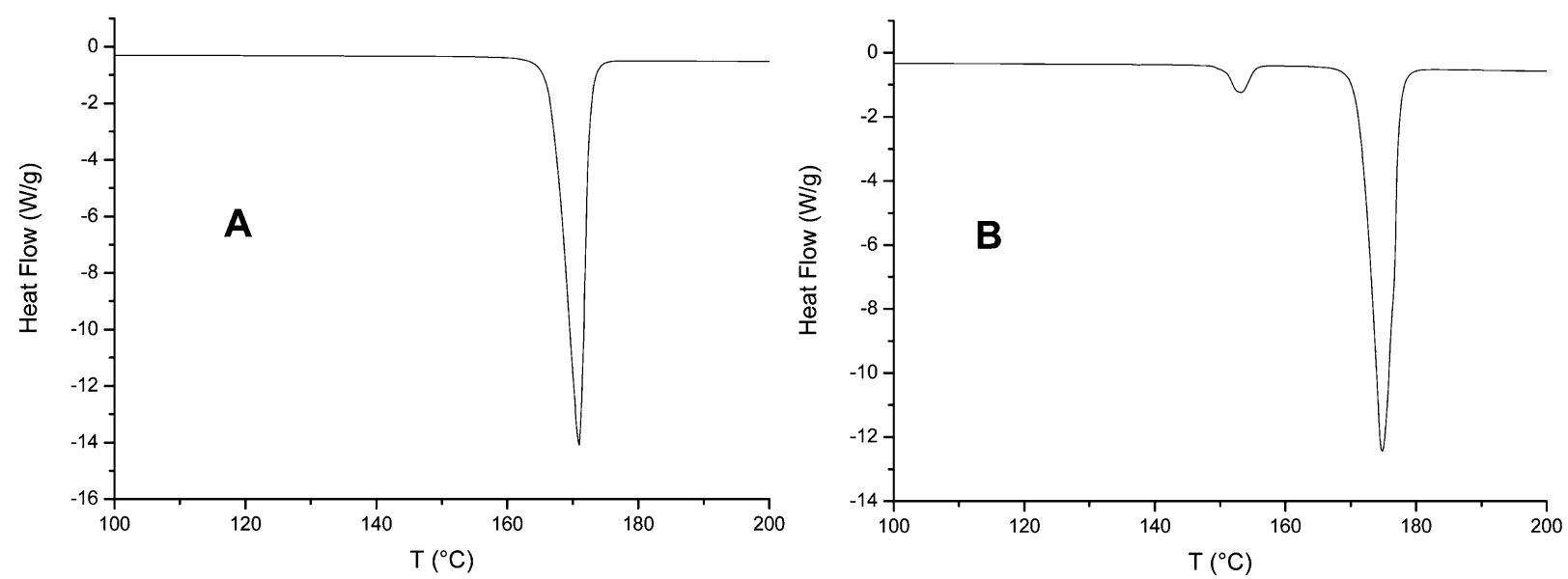

Figure 1. Weight-normalized DSC thermograms of monoclinic malonamide. (A) Direct melting and (B) monoclinic toward quadratic transition prior to the quadratic phase melting.

Table 1. Temperatures, Enthalpies, and Entropies of the Transition Points of Malonamide (Stable and Metastable (*))

\begin{tabular}{|c|c|c|c|c|c|}
\hline & \multicolumn{3}{|c|}{ melting points } & \multirow[b]{2}{*}{ monoclinic-quadratic transition } & \multirow[b]{2}{*}{ ref } \\
\hline & orthorhombic* & monoclinic* & quadratic & & \\
\hline \multirow[t]{3}{*}{$T(\mathrm{~K})$} & $431.06 \pm 0.06$ & $439.49 \pm 0.06$ & $443.98 \pm 0.06$ & $423.50 \pm 0.06$ & this work \\
\hline & 435 & & 443 & 393 & 4 \\
\hline & $433.66-436.78$ & & $445.09-446.53$ and $444.59-445.48$ & $408.48-292.21$ & 7 \\
\hline \multirow[t]{3}{*}{$\Delta H\left(\mathrm{~kJ} \cdot \mathrm{mol}^{-1}\right)$} & $24.2 \pm 0.5$ & $30.3 \pm 0.4$ & $28.8 \pm 0.2$ & $1.66 \pm 0.04$ & this work \\
\hline & 29.8 & & 35.8 & 1.9 & 4 \\
\hline & 30.24 & & 24.8 and 31.1 & 1.49 & 7 \\
\hline$\Delta S\left(\mathrm{~J} \cdot \mathrm{mol}^{-1} \cdot \mathrm{K}^{-1}\right)$ & 56.1 & 69.9 & 64.9 & 3.9 & this work \\
\hline
\end{tabular}
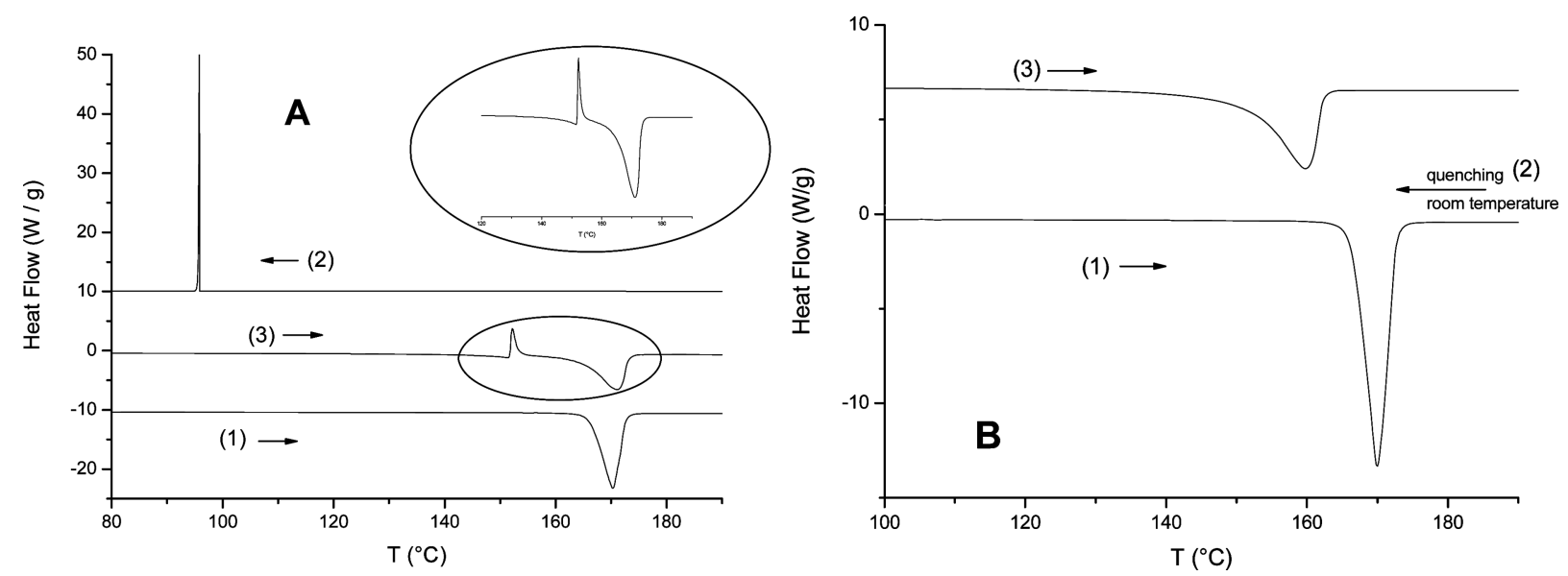

Figure 2. Weight-normalized DSC thermograms of orthorhombic malonamide. (A) Melting-recrystallization phenomenon after a heating-cooling cycle and (B) direct melting after quenching the melt at room temperature.

Cell parameters were refined by pattern matching using the freely crystallographic program JANA2006. ${ }^{9}$ The background was fitted with a Legendre polynomial. The pseudo-Voigt profile function was used for describing the shape of diffraction peaks. The initial parameters were taken from the CIF files of the different phases of the malonamide compound. ${ }^{3,5,6}$

Solubility Measurements. Malonamide powder was dissolved in water until saturation at different temperatures in a temperature-controlled device, with a precision of $\pm 1{ }^{\circ} \mathrm{C}$. At a given temperature, a part of the supernatant saturated solution was withdrawn by means of a syringe and then placed in a sealed preweighed container $\left(m_{\mathrm{s}}\right)$. After that, the container was opened for total water evaporation. The dry powder was then weighed $\left(m_{\mathrm{p}}\right)$. The solubility $s$ of monoclinic malonamide was determined for each temperature by eq 1 :

$$
s=\frac{m_{\mathrm{p}}}{m_{\mathrm{s}}-m_{\mathrm{p}}}
$$

where $\left(m_{\mathrm{s}}-m_{\mathrm{p}}\right)$ represents the mass of water. The crystal structure of the dry powder was controlled by X-ray powder diffraction.

\section{RESULTS AND DISCUSSION}

Although the monoclinic phase is stable at ambient conditions up to $\sim 150^{\circ} \mathrm{C}$ prior to transiting into the quadratic one, ${ }^{4,7}$ no solid modification was observed on heating the commercial product. 
In that case, the monoclinic phase melted directly (Figure 1A). This singular behavior allowed us to obtain the melting characteristics of the corresponding monoclinic phase (Table 1). It is noteworthy that, although the enantiotropic behavior between the monoclinic and quadratic phases is a reversible process, the transition is, in this case, bypassed. As a matter of fact, the monoclinic toward quadratic transition was noticed only after the monoclinic phase milling, as shown in Figure 1B.

The orthorhombic phase was obtained either by quenching at room temperature or by cooling down with controlled temperature the molten commercial monoclinic phase. However, upon heating the orthorhombic polymorph, a random thermal behavior had been noticed, due to either partial melting and recrystallization in the monoclinic phase before its own melting (Figure 2A, curve 3) or direct melting (Figure 2B, curve 3 ).

The powder of the three solid forms was analyzed by X-ray powder diffraction. The diffraction patterns of monoclinic and quadratic phases were recorded at temperatures where the phases are stable $\left(25\right.$ and $165{ }^{\circ} \mathrm{C}$ respectively). The orthorhombic polymorph was obtained by quenching liquid malonamide at room temperature. Unfortunately, the X-ray diffraction pattern of pure orthorhombic phase was never obtained since the phase transformed with time in tetragonal phase. However, by taking into account the only diffraction peaks of the orthorhombic phase, a refinement was made possible. The refinement of the $\mathrm{X}$-ray diffraction patterns for the three polymorphs was only a pattern matching without structural model. This allowed us to obtain the fitted lattice parameters as a function of temperature. The patterns are presented in Figure 3 and were found to be the same as the profiles calculated according to the crystal structures. ${ }^{3,5,6}$

It is noteworthy that, once malonamide is molten, the reversible liquid toward quadratic transition cannot be formed. In such a case, the orthorhombic or the monoclinic form is obtained. This necessarily implies that the thermodynamic reversible quadratic toward monoclinic transition is not possible either. Since the thermodynamic considerations indicate that the transitions are reversible, the kinetic contribution should be taken into account. For that purpose, the kinetics of transformation of the orthorhombic phase was followed by X-ray diffraction experiments at room temperature. We demonstrated that the orthorhombic form transits to the monoclinic one after approximately 20 days (result not shown). Although this result is obvious, it reinforces the idea that the orthorhombic phase is less stable than the monoclinic one at room temperature. This was corroborated by the increase of the melting temperatures from the orthorhombic to the monoclinic system (Figure 4). When the two polymorphs are monotropes of each other, the following rule applies: "the higher the melting point, the higher the relative stability". Consequently, the monoclinic phase is always stable below the melting point of both polymorphs.

As far as the quadratic phase is concerned, one can see from Figure 4 that this phase has the highest melting point. This is in accordance with the fact that, above the temperature of the solid-solid transition point, it is the most stable phase. Furthermore, from the DSC results presented in Figure 4, the temperatures and enthalpies of melting of the quadratic and monoclinic phases, as well as of the monoclinic toward quadratic solid-solid transition, were estimated and compared to the literature values (Table 1). The data gathered in Table 1 show some discrepancies for all reported values. However, owing to
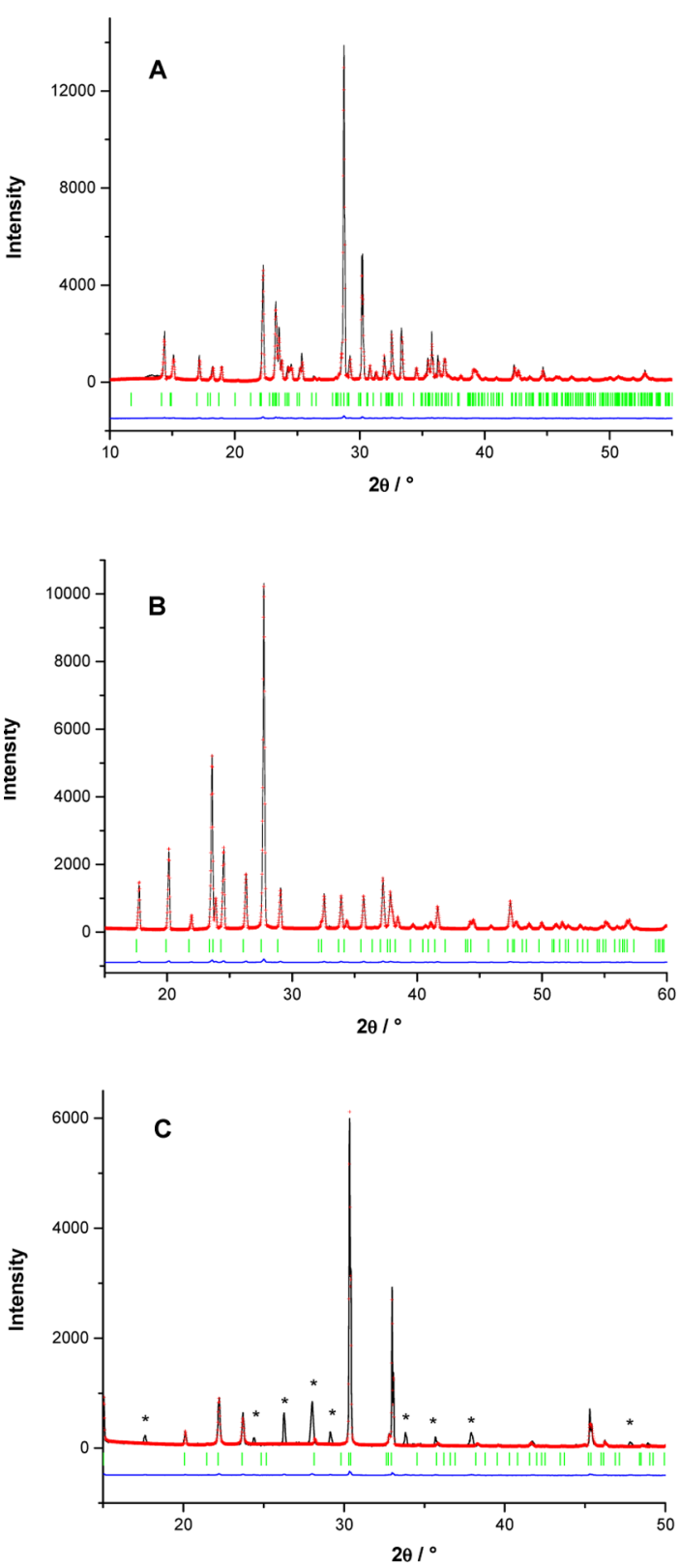

Figure 3. X-ray diffraction patterns and their refinement for the three polymorphs of malonamide: (A) monoclinic $\left(25^{\circ} \mathrm{C}\right)$; (B) tetragonal $\left(165^{\circ} \mathrm{C}\right)$; $(\mathrm{C})$ orthorhombic $\left(25^{\circ} \mathrm{C}\right)$. Solid black line $=$ experimental pattern; red crosses $=$ calculated pattern; blue line $=$ difference between the observed and the calculated patterns; vertical green bars = peak positions. The asterisks in $\mathrm{C}$ indicate the diffraction peaks relative to the tetragonal phase present in the orthorhombic pattern.

this work, the melting properties of the monoclinic form are proposed for the first time.

The low value of entropy for the solid-solid transition (6\% of the melting enthalpy of the quadratic phase, Table 1) may explain why, on heating the monoclinic phase, the transition, although reversible, does not take place.

However, one cannot be sure that complete recrystallization into the monoclinic phase is achieved. Consequently, the melting heat of the recrystallized phase might be underestimated. In order to answer such a question, a Hess cycle can be used, taking into account the solid-solid transition, the melting point of the quadratic phase, and the experimentally determined $C_{p}$ values of the phases involved (Table 2). 


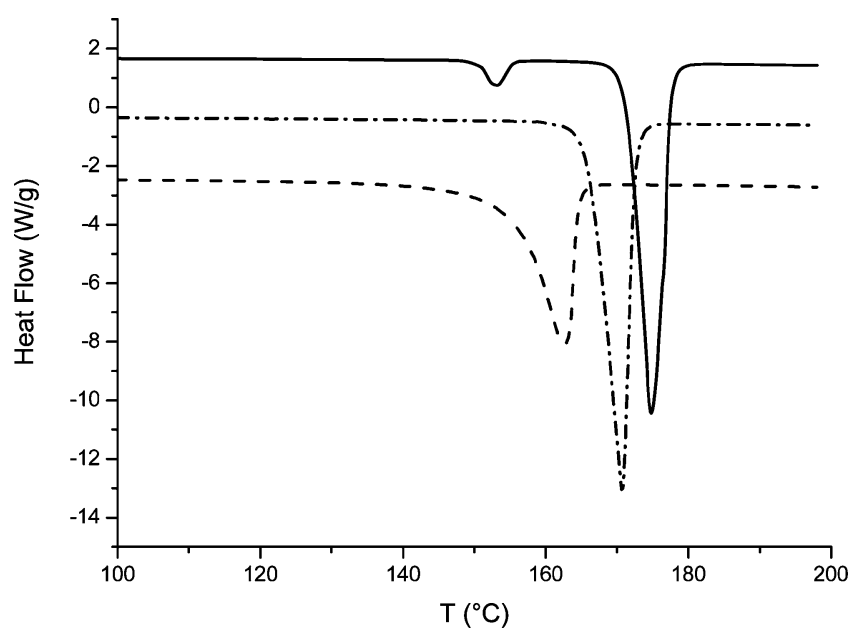

Figure 4. Weight-normalized DSC thermograms presenting the melting peaks of the three forms of malonamide: orthorhombic (dashed line), monoclinic (dash-dotted line), quadratic (solid line).

Table 2. Coefficients of Regression $a$ and $b$ for $C_{p}\left(\mathrm{~J} \cdot \mathrm{mol}^{-1}\right.$. $\left.\mathrm{K}^{-1}\right)=a T(\mathrm{~K})+b$, and Their Corresponding Standard Deviations

\begin{tabular}{lllc} 
& $a\left(\mathrm{~J} \cdot \mathrm{mol}^{-1} \cdot \mathrm{K}^{-2}\right)$ & $b\left(\mathrm{~J} \cdot \mathrm{mol}^{-1} \cdot \mathrm{K}^{-1}\right)$ & $r^{2}$ \\
orthorhombic & $0.609 \pm 0.007$ & $-33 \pm 3$ & 0.98898 \\
monoclinic & $0.45 \pm 0.05$ & $5 \pm 2$ & 0.99291 \\
quadratic & $5.1 \pm 0.3$ & $-2044 \pm 135$ & 0.98217 \\
liquid & $0.59 \pm 0.01$ & $2 \pm 4$ & 0.99141 \\
\hline
\end{tabular}

As for the monoclinic phase $\mathrm{M}$, the assumed melting temperature is $439.49 \pm 0.06 \mathrm{~K}$, and the corresponding heat of fusion can be obtained as follows:

$$
\begin{aligned}
& \Delta_{\text {fus }} H(\mathrm{M}, 439.49 \mathrm{~K}) \\
& \quad=\Delta_{\text {trans }} H(\mathrm{M}-\mathrm{Q} 439.49 \mathrm{~K})+\Delta_{\text {fus }} H(\mathrm{Q} 439.49 \mathrm{~K})
\end{aligned}
$$

with

$$
\begin{aligned}
& \Delta_{\text {trans }} H(\mathrm{M}-\mathrm{Q} 439.49 \mathrm{~K}) \\
& =\Delta_{\text {trans }} H(\mathrm{M}-\mathrm{Q} 423.50 \mathrm{~K})-\int_{423.50}^{439.49} C_{p, \mathrm{M}} \mathrm{d} T \\
& \quad+\int_{423.50}^{4439.49} C_{p, \mathrm{Q}} \mathrm{d} T \\
& \Delta_{\text {fus }} H(\mathrm{Q} 439.49 \mathrm{~K}) \\
& =\Delta_{\text {fus }} \mathrm{H}(\mathrm{Q} 443.98 \mathrm{~K})+\int_{439.49}^{443.98} C_{p, \mathrm{Q}} \mathrm{d} T \\
& \quad-\int_{439.49}^{443.98} C_{p, \mathrm{~L}} \mathrm{~d} T
\end{aligned}
$$

Taking into account the $C_{p}$ values from Table 2, $\Delta_{\text {fus }} H(\mathrm{M}, 439.49 \mathrm{~K})$ was found, from eq 2 , to be equal to 29.6 $\pm 0.6 \mathrm{~kJ} \cdot \mathrm{mol}^{-1}$.

The enthalpy value obtained by calculus compares well with the value of $30.3 \pm 0.4$ found experimentally. This result confirms that the monoclinic phase completely recrystallizes before melting in the conditions described above.

Another approach has been implemented experimentally in order to determine the melting enthalpy of the monoclinic phase. Since this phase is the one which is stable up to $150.5^{\circ} \mathrm{C}$

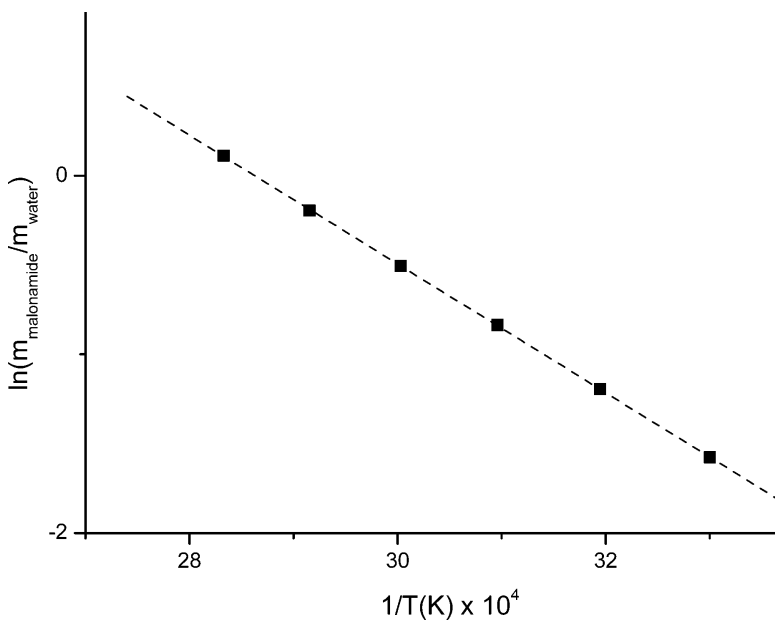

Figure 5. Van't Hoff type plot: the monoclinic malonamide solubility in water vs the inverse of temperature $T$ in kelvins.

$(423.5 \mathrm{~K})$, solubility measurements in water were carried out from 30 to $85{ }^{\circ} \mathrm{C}$. A Van't Hoff type plot, given the Napierian logarithm of the ratio of the masses of solid malonamide and water as a function of the inverse of the temperature in kelvins, is shown in Figure 4. A linear relation is thus obtained, implying the following equation (eq 3 ):

$$
\ln (x)=\frac{-\Delta_{\text {fus }} H}{R} \times \frac{1}{T}
$$

From the fitting of the data plotted in Figure 5, one can deduce, using eq 3 , that

$$
\begin{aligned}
\ln (x) & =(-0.0360 \pm 0.001) \times \frac{1}{T}+(10.32 \pm 0.04) \\
\left(r^{2}\right. & =0.99995)
\end{aligned}
$$

From the above result, the heat of fusion for the monoclinic form is found equal $29.93 \pm 0.01 \mathrm{~kJ} \cdot \mathrm{mol}^{-1}$. This result also reinforces the method used to determine this quantity from the Hess cycle, as calculated above.

The "heat of fusion rule" states that "if the higher melting form has the lower heat of fusion, then the two polymorphs are usually enantiotropic". ${ }^{10}$ Applying this rule to the case of malonamide, and more specifically to each combination of the three polymorphs, we get that the monoclinic phase and the quadratic one are enantiotropically related since the quadratic form melts with a higher melting point and with a lower heat of fusion. On the contrary, if we compare the monoclinic and the quadratic phases, although the quadratic phase melts with the higher melting point, its associated melting enthalpy is higher than the monoclinic one. In that case, it can be deduced that the two phases are monotropically related. The same conclusion is drawn for the relation between the monoclinic and the orthorhombic forms.

However, it has to be noticed that these interpretations are valid if we consider a constant pressure when the polymorphic forms are heated. This pressure, which can be assumed to be close to the vapor pressure of the substance at a given temperature, has to be considered as "low pressure" by opposition to "high pressure." This latter case reflects the case in which the system undergoes a high external pressure.

The relative thermodynamic stability of the solid phases can be apprehended through the density rule enunciated by 
Kitaigorodskii, ${ }^{11}$ and resumed by Burger for "pharmaceuticals and other molecular crystals", ${ }^{10}$ that states that the crystal packing is conditioned by the shortest distances between the atoms of adjacent molecules. Even though this principle is assumed to be general for molecular solids dominated by van der Waals interactions, one cannot exclude to apply it to systems involving hydrogen bonds such as malonamide polymorphs. This is the reason why $\mathrm{X}$-ray powder diffraction experiments as a function of the temperature have been performed for the three polymorphs in order to determine their specific volumes $\mathrm{V} / \mathrm{m}$ (Figure 6). Standard deviations from these calculations are also plotted in Figure 6. As a matter of fact, the specific volumes $\mathrm{V} / \mathrm{m}$ of malonamide polymorphs were previously determined for only
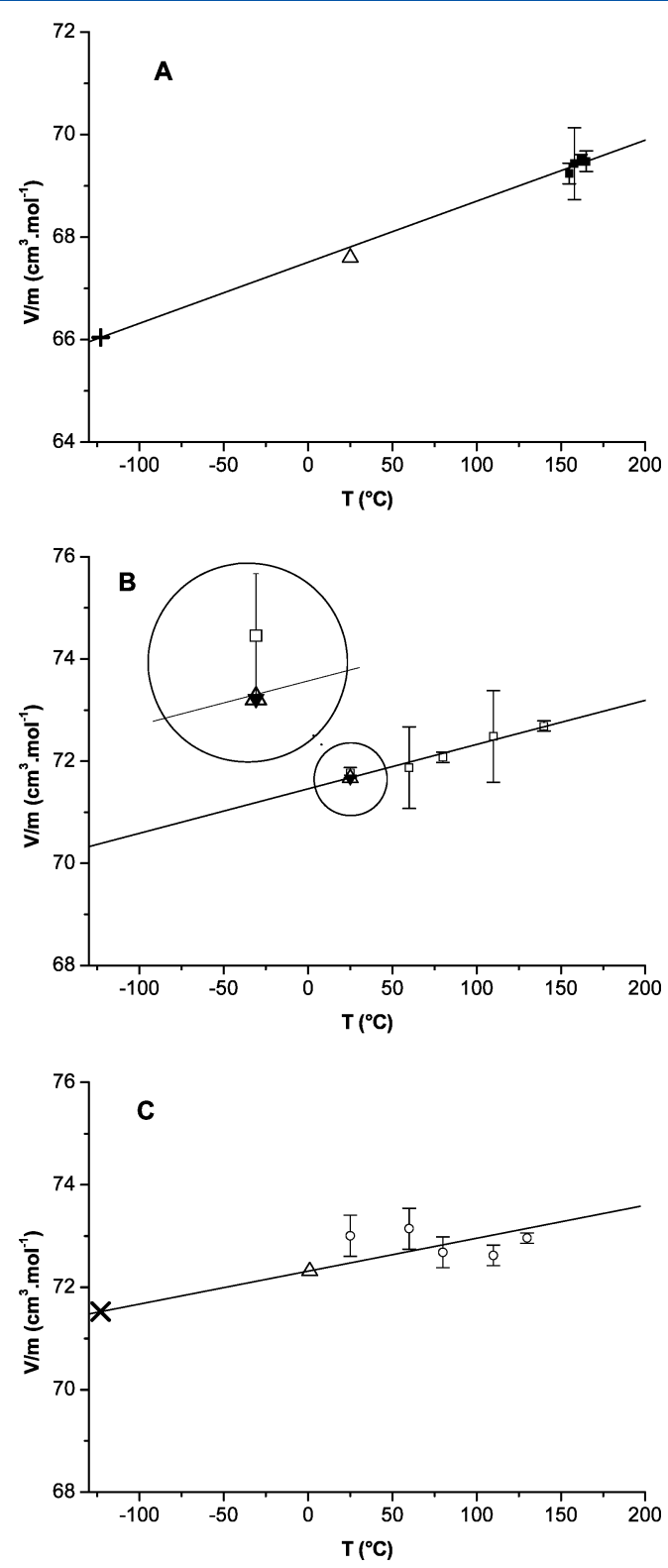

Figure 6. Experimental specific volumes vs temperature for the three solid forms of malonamide: (A) quadratic ( $\square$ ), (B) monoclinic ( $\square$ ), and (C) orthorhombic $(\mathrm{O})$. The data obtained from the literature are represented by + (quadratic form), $\boldsymbol{\nabla}$ (monoclinic form), and $\times$ (orthorhombic form), from refs 35 , and 6, respectively. The three polymorph specific volumes from ref 7 are represented by $\Delta$. The inset in $\mathrm{B}$ helps differentiate more clearly the data points. few temperatures. More specifically, only two data sets were found in the literature which, in addition, were determined at one and the same temperature for the monoclinic polymorph (ambient temperature). The supplementary data points obtained from the present study allowed determining the evolution of $V /$ $m$ as a function of the temperature. Our results, compared to the data obtained from the literature, show a good correlation. The parameters obtained from the specific volume fittings are reported in Table 3.

Table 3. Parameters Obtained from the Malonamide Specific Volume Fittings in Its Solid State as a Function of Temperature in $\mathrm{K}^{a}$

\begin{tabular}{cccl} 
& $\mathrm{a}$ & $\mathrm{b}$ & \multicolumn{1}{c}{$\mathrm{r}^{2}$} \\
orthorhombic & 72.377 & $5.16706 \times 10^{-5}$ & 0.637 \\
monoclinic & 71.378 & $9.20045 \times 10^{-5}$ & 0.9467 \\
quadratic & 67.420 & $1.19959 \times 10^{-1}$ & 0.99275 \\
${ }^{a} V / m\left(\mathrm{~cm}^{3} \cdot \mathrm{mol}^{-1}\right)=a+b \times T$. & & \\
\hline
\end{tabular}

As it can be noticed, the density of the orthorhombic polymorph is always lower than that of the other two polymorphs. This can also be explained by the monotropic relation established between the orthorhombic phase and one of the other two phases. Despite the fact that packing of the molecules in the solid state is governed by hydrogen bonding, the conclusion is the same as the one coming from the density rule. Regarding the quadratic phase, it is denser than the monoclinic form while the two phases are enantiotropes. In that case, the density rule does not apply.

A thermodynamic approach to calculating the Gibbs energies was also apprehended based on the theoretical model developed by $\mathrm{Yu}^{12}$ Assuming a constant pressure close to the vapor pressure of the substance (the transition data are obtained on heating malonamide under its own vapor pressure), one can estimate the Gibbs energy difference between two polymorphs of malonamide, for instance, between the quadratic and the monoclinic phases $(\Delta G(T)=G($ quadratic $)-G($ monoclinic $))$, which are enantiotropically related. This model allows inferring the thermodynamic stability relation of polymorphs from only melting data.

Assuming a nonlinearity behavior for $\Delta G$ as a function of temperature in kelvins, we get eq 4:

$$
\begin{aligned}
\Delta_{\text {trans }} & G(\mathrm{M}-\mathrm{Q}, T) \\
= & \Delta_{\text {fus }} G(\mathrm{M}, 439.49 \mathrm{~K})-\Delta_{\text {fus }} S(\mathrm{M}, 439.49 \mathrm{~K}) \\
& \times\left(T-T_{\text {fus }}(\mathrm{M})\right) \\
& -\left(\mathrm{C}_{p, \mathrm{Q}}-C_{p, \mathrm{M}}\right) \times \frac{\left(\left(T-T_{\mathrm{fus}}(\mathrm{M})^{2}\right)\right.}{2 T_{\text {fus }}(\mathrm{M})}
\end{aligned}
$$

with

$$
\begin{aligned}
& \Delta_{\text {fus }} G(\mathrm{M}, 439.49 \mathrm{~K})=\Delta_{\text {fus }} H(\mathrm{Q}) \times\left(\frac{T_{\text {fus }}(\mathrm{M})}{T_{\text {fus }}(\mathrm{Q})}-1\right) \\
& +\left(\mathrm{C}_{p, \mathrm{~L}}-C_{p, \mathrm{Q}}\right) \times\left[T_{\text {fus }}(\mathrm{Q})-T_{\text {fus }}(\mathrm{M})\right. \\
& \left.-T_{\text {fus }}(\mathrm{M}) \times \ln \frac{T_{\text {fus }}(\mathrm{Q})}{T_{\text {fus }}(\mathrm{M})}\right]
\end{aligned}
$$

and 


$$
\begin{aligned}
& \Delta_{\text {fus }} S(\mathrm{M}, 439.49 \mathrm{~K}) \\
& =\frac{\Delta_{\text {fus }} H(\mathrm{M}, 439.49 \mathrm{~K})}{T_{\text {fus }}(\mathrm{M})}-\frac{\Delta_{\text {fus }} H(\mathrm{Q}, 443.98 \mathrm{~K})}{T_{\text {fus }}(\mathrm{Q})} \\
& \quad+\left(\mathrm{C}_{p, \mathrm{~L}}-C_{p, \mathrm{Q}}\right) \times \ln \frac{T_{\text {fus }}(\mathrm{Q})}{T_{\text {fus }}(\mathrm{M})}
\end{aligned}
$$

The heat capacities, $C_{p, \mathrm{~L}}, C_{p, \mathrm{Q}}$, and $C_{p, \mathrm{M}}$ are determined at $T_{\text {fus }}(\mathrm{M})$, using the $C_{p}$ data given in Table 2 .

Then, eq 4 becomes

$$
\begin{aligned}
\Delta_{\text {trans }} G(\mathrm{M}-\mathrm{Q} T) \\
=-289.8-4.725 \times(T-439.49)-(197.4-202.8) \\
\quad \times \frac{(T-439.49)^{2}}{2 \times 439.49}
\end{aligned}
$$

The resulting plot is a second order polynomial which decreases with temperature from positive values to negative ones with a zero-value obtained at $373 \mathrm{~K}$. That confirms the enantiotropic behavior between the quadratic and the monoclinic phases. Below the experimental transition point (i.e., $423.5 \mathrm{~K}$ ), the monoclinic phase is stable $\left(G_{\text {monoclinic }}<G_{\text {quadratic }}\right)$, and above the transition point, the quadratic phase turns to be stable $\left(G_{\text {quadratic }}<G_{\text {monoclinic }}\right)$ before melting. Due to approximations used for calculation, the transition temperature obtained this way is quite different compared to the experimental value. It is nevertheless true that the relative thermodynamic stability is perfectly established between the quadratic and the monoclinic forms.

The same thermodynamic approach was adopted to compare the relative stability between the monoclinic and the orthorhombic forms. The following equation (eq 5) was obtained:

$$
\begin{aligned}
& \Delta_{\text {trans }} G(\mathrm{O}-\mathrm{M}, T) \\
&= 11428.4-11.692 \times(T-431.06) \\
& \quad-(198.977-226.106) \times \frac{(T-431.06)^{2}}{2 \times 431.06}
\end{aligned}
$$

The energy values decrease continuously when the temperature increases but remain positive. This goes along with the monotropic behavior between the orthorhombic and the monoclinic phases, which confirms that the orthorhombic phase is always less stable than the monoclinic one.

Interestingly, if we compare the relative stability between the quadratic and the orthorhombic forms, the Gibbs energy values are positive up to approximately $310 \mathrm{~K}$, and then they become negative. This implies that, between the two metastable phases, relative to the monoclinic phase, the quadratic form is "more metastable" than the orthorhombic one for temperatures below $310 \mathrm{~K}$. This hierarchy is reversed at higher temperatures up to $373 \mathrm{~K}$ (423.5 K experimentally). Above this temperature, the quadratic form becomes the stable phase up to melting.

At $310 \mathrm{~K}$, when the Gibbs energy difference equals zero, we are at the equilibrium point where the two phases (orthorhombic and quadratic) coexist, in equilibrium with the vapor phase (triple point). This triple point is obviously virtual since it is a metastable point.

From the previous calculations, at low temperatures, one can propose that (i) the quadratic phase is more stable than the orthorhombic and the monoclinic ones, and (ii) the orthorhombic phase is more stable than the monoclinic one.
Increasing the $G$ values, we necessarily have, at a given temperature, the following hierarchy:

$$
\text { monoclinic } \rightarrow \text { orthorhombic } \rightarrow \text { quadratic }
$$

These thermodynamic inferences about the relative stabilities of the polymorphs allow us to propose the energy-temperature diagram presented in Figure 7.

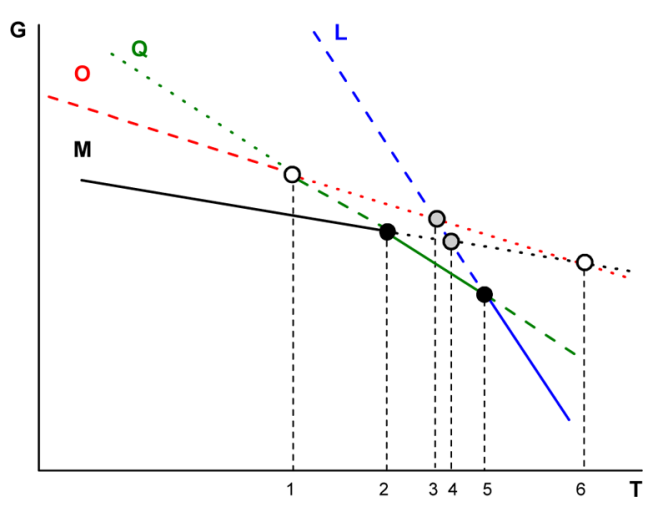

Figure 7. Schematic Gibbs energy diagram as a function of the temperature representing the Gibbs energy of the three solid forms $(\mathrm{Q}$ $\mathrm{O}$, and $\mathrm{M}$ ) and the liquid phase (L). Solid lines: Gibbs energy of the stable phases. Dashed lines: Gibbs energy of the phases in a metastable state (higher free energy). Dotted lines: Gibbs energy of the phases in a lower metastable state (even higher energy). 1: metastable solid-solid transition point between the orthorhombic $(\mathrm{O})$ and the quadratic $(\mathrm{Q})$ phases. 2: stable solid-solid transition point between the monoclinic (M) and the quadratic (Q) phases. 3: Metastable orthorhombic melting point. 4: Metastable monoclinic melting point. 5: Stable quadratic melting point. 6: Extrapolated metastable solid-solid transition point between the orthorhombic $(\mathrm{O})$ and the monoclinic $(\mathrm{M})$ phases.

As the diagram shows, the melting temperatures of the solid forms increase with their relative stability (points 3 to 5 in Figure 7), and agree well with the experiments performed. As observed in Figure 7, the Gibbs energy curves of the monoclinic and quadratic phases have to converge at high temperature (point 5 in Figure 7). From the calculations, this point is predicted at $475 \mathrm{~K}$. It corresponds to the triple point where the two solid phases are in equilibrium with the vapor phase.

\section{CONCLUSION}

The relative stability of the three crystal modifications of malonamide is described in terms of Gibbs energy calculations corroborated by the experimental part achieved on the three polymorphs. The monotropic and enantiotropic nature between the three solids has been fully elucidated. The malonamide Gibbs free energy-temperature diagram established in this study clearly shows the following: (i) The orthorhombic phase is actually monotrope with respect to the two other solid phases. (ii) The solid-solid phase transition between the orthorhombic and the tetragonal phases takes place at low temperature but remains metastable since it occurs at a higher energy level than the one of the monoclinic phase. This point clearly contradicts the assumption made in ref 7 , which stated that this transition point takes place at high temperature, i.e., above the melting point of the quadratic form. (iii) The virtual phase transition between the orthorhombic and the monoclinic phases occurs at high temperature. The only study of malonamide phase transitions by thermal analysis did not allow locating this point. ${ }^{7}$ 
However, the thermodynamic approach developed here for determining the hierarchy of stability of the polymorphs is very sensitive to changes in $C_{p}$. This may explain why the calculated temperature of the monoclinic-quadratic transition slightly differs from that found experimentally. But, fortunately, the accuracy of the calculation has no effect on the relative stabilities. It appears that the experimental approach by means of thermal analysis and X-ray diffraction coupled with the thermodynamic approach leads to a coherent explanation of the hierarchy of stability of the three polymorphs of malonamide under ordinary conditions of pressure. However, this approach obscures the influence of pressure on the possible polymorphic transformations. This variable is of crucial importance when we deal with the dosage form of a drug. In this case, it would be wise to determine the state diagram of the substance involving the two fundamental variables, temperature and pressure.

\section{AUTHOR INFORMATION}

\section{Corresponding Author}

*Tel: 33153739676. E-mail: philippe.espeau@parisdescartes.fr.

\section{Notes}

The authors declare no competing financial interest.

\section{ACKNOWLEDGMENTS}

This work was supported in part by the Agence Nationale de la Recherche under the NPLIN-4-Drug project. The authors thank L. Ferrand and A. Hautier, as MSc and BSc students, respectively, for their contribution to this work. K. Debbasch is also thanked for fruitful discussions on the manuscript.

\section{REFERENCES}

(1) Follmann, M.; Kirsch, R.; Lorenz, M.; Matter, H.; Neuenschwander, W. K.; Scotese, C. A.; Steinhagen, H.; Szillat, H.; Wehner, V. Malonamide derivatives with antithrombotic activity, Patent WO2009007015A1, January 15, 2009.

(2) Kostlan, C. R.; Raheja, R. K.; Tugnait, M.; Wade, K. J. Pharmaceutical compositions comprising malonamide derivatives for decreasing sebum production. Patent WO2005034931A1, April 15, 2005.

(3) Chieh, P. C.; Subramanian, E.; Trotter, J. Crystal Structure of Malonamide. J. Chem. Soc. A 1970, 179-184.

(4) Sakiyama, M.; Imamura, A. Thermoanalytical Characterization of 1,3-Dimethyluracil and Malonamide Crystals. Thermochim. Acta 1989, 142, 365-370.

(5) Nichol, G. S.; Clegg, W. Malonamide: an orthorhombic polymorph. Acta Crystallogr. 2005, E61, o3424-o3426.

(6) Nichol, G. S.; Clegg, W. Malonamide: a tetragonal polymorph. Acta Crystallogr. 2005, E61, o3427-o3429.

(7) Cheung, E. Y.; Peterson, M. L. Reconciling Thermal and Structural Data From The Polymorphic Transitions of Malonamide. Thermochim. Acta 2009, 482, 57-61.

(8) Lazerges, M.; Corvis, Y.; Pardieu, E.; Bouteiller, L.; Espeau, P. Fluid Phase Equilib. 2012, 315, 107-112.

(9) Petricek,V.; Dusek, M.; Palatinus, L. Jana2006: Program for structure analysis of crystals periodic in three or more dimensions from diffraction data (http://www-xray.fzu.cz/jana); Institute of Physics: Praha, Czech Republic, 2006.

(10) Burger, A.; Ramberger, R. On the polymorphism of pharmaceuticals and other molecular compounds. Part I: Theory of thermodynamic rules. Microchim. Acta [Wien] 1979, 259-271.

(11) Kitaigorodskii, A. I. Molecular crystals and molecules; Academic Press: New York, 1973.

(12) Yu, L. Inferring Thermodynamic Stability Relationship of Polymorphs from Melting Data. J. Pharm. Sci. 1995, 84, 966-974. 